\title{
Correspondence
}

\section{Friday the 13th and fatal self-harm}

Sir: The belief that Friday the 13th is unlucky for some is entrenched in our language and has been held throughout history in many cultures. Scanlon et al (1993) reported that the risk of hospital admissions as a result of a transport accident may be increased by as much as $52 \%$ on Friday the 13th. They recommended staying at home.

Superstitiousness correlates significantly with some aspects of compulsive behaviour and obsessional ruminations (Veale, 1995), and superstitious people see causal relationships between their own thoughts or actions and events in the world. Superstitions, such as unlucky Friday the 13th, may affect our behaviour and tend to flourish whenever we cannot tolerate uncertainty or believe that we have no control over events. The presence or absence of an association between events such as suicide and Friday the 13th does not appear to have been reported previously.

Between 1989 and 1995 there were 364 Fridays, 84 13th days of the month and 12 were Friday the 13th. A total of 277 incidents of fatal self-harm (FSH) were reported within North Cheshire. (The term FSH is used here to describe all coroners' verdicts of suicide, misadventure and open verdict.) Fifty FSH incidents were reported on all Fridays (13.7\%), 10 on the 13th days $(11.9 \%)$, one incident on Friday the 13th and one on Friday the 6 th $(8.3 \%)$. There is no statistically significant difference between proportions $\left(\chi^{2}\right.$ : d.f. $\left.=3, P>0.05\right)$ indicating that FSH is equally likely to occur on Friday the 13 th as on Friday the 6th (the day chosen by Scanlon et al, 1993 for comparison), as well as on any Friday and any 13th day. Based on the small numbers available for this review, the probability of an incident of FSH occurring or being reported on Friday the 13th or Friday the 6th was 0.004 (one incident in 277), identical to the proportion of either Friday 13th or 6th in the seven years of data collection (12 days in 2555). This means that FSH reported in North Cheshire on Friday the 13th probably occurred by chance alone and provides no evidence of any increased or reduced risk on this particular day. There was also no observed pattern on the day before or after Friday the 13th. Mean psychiatric admissions did not differ significantly on those days except for a generally reduced rate of hospital admissions on the 13th day over the same period.

All days are certainly unlucky days for those who succeed in harming themselves fatally, deliberately or otherwise, and for the unfortunate people they leave behind. However, Friday the 13th appears to have been neither chosen nor avoided.

SCANLON, T. J., LUBEN, R. N., SCANLON, F. L., et al (1993) Is Friday the 13th bad for your health? British Medical Joumal, 307, 1584-1586.

VEALE. D. (1995) Friday the 13th and obsessive compulstve disorder. British Medical Journal, 311, 863-864.

EMAD SAlib, Nicola GRAY and MARK SPYE, Winwick Hospital, Warrington

\section{Objective structured clinical examination}

Sir: The Nottingham report on objective structured clinical examination (OSCE) (Psychiatric Bulletin, January 1997, 21, 30-32) fuels interest in undergraduate assessment methods and is a veritable response to the General Medical Council's call for innovativeness in course objectives and structuring. However, the traditional long case (TLC) has more demerits than was portrayed. It is true that the TLC presents opportunity for assessing interactional skills, and a candidate can rectify an error made earlier in the examination. But in real life practice some avoidable errors cannot be rectified.

From our modest experience, we believe that surreptitiously set OSCE stations could test interpersonal skills to an acceptable degree. We should furthermore emphasise that fairness, objectivity (minimal examiner's bias), an ingredient of validity, wide coverage of the course and economy of examining time are the unique virtues of the OSCE.

Comparability of examination methods is difficult, especially with variability of settings and instructional objectives (Petrusa et al, 1986); however, a holistic examination package which retains the TLC in an attenuated form offers bright prospects of meaningful assessment of students.

Petrusa, E. R., Blackwell, T. A., Parcel, S. L., et al (1986) Psychometric properties of the objective clinical examination (OSCE) as an instrument for final evaluation. In Newer Developments in Assessing Clinical Competence (eds I. R. Hart, R. M. Harden \& 